$5-23-1994$

\title{
Scaling Behavior of Fluctuations in Systems with Continuous Symmetry
}

Ulrich Zürcher

Cleveland State University, u.zurcher@csuohio.edu

Follow this and additional works at: https://engagedscholarship.csuohio.edu/sciphysics_facpub

Part of the Physics Commons

How does access to this work benefit you? Let us know!

Publisher's Statement

(C)1994 American Physical Society

\section{Repository Citation}

Zürcher, Ulrich, "Scaling Behavior of Fluctuations in Systems with Continuous Symmetry" (1994). Physics Faculty Publications. 278.

https://engagedscholarship.csuohio.edu/sciphysics_facpub/278

This Article is brought to you for free and open access by the Physics Department at EngagedScholarship@CSU. It has been accepted for inclusion in Physics Faculty Publications by an authorized administrator of

EngagedScholarship@CSU. For more information, please contact library.es@csuohio.edu. 


\title{
Scaling Behavior of Fluctuations in Systems with Continuous Symmetry
}

\author{
Ulrich Zürcher \\ Department of Chemistry, Massachusetts Institute of Technology, Cambridge, Massachusetts 02139
}

(Received 22 December 1993)

\begin{abstract}
In nematic liquid crystals, director fluctuations correspond to the dynamical mode that is critical for all temperatures in zero external fields. The Hurst exponent characterizes the temporal behavior of the mean square displacement of director fluctuations, $\left\langle[\delta n(r, t)-\delta n(r, 0)]^{2}\right\rangle \sim t^{2 H}$. We find $H=\frac{1}{2}$ in finite fields and $H=\frac{3}{4}$ in zero field. This result differs from that of Zhang et al. [Phys. Rev. Lett. 70, 1834 (1993)] who find a Hurst exponent that varies continuously from $H \simeq 1$ in zero field to $H \simeq \frac{1}{2}$ in moderate fields.
\end{abstract}

PACS numbers: $64.70 . \mathrm{Md}, 05.40 .+\mathrm{j}, 61.30 .-\mathrm{v}, 64.60 . \mathrm{Cn}$

In situations where the dynamic produces its own ordering, the term "self-organized criticality" has been proposed by Bak, Tang, and Wiesenfeld [1]. Such behavior has been studied extensively in sandpile models [2], where the total number of particles is conserved and the average density of particles per site is the associated "order parameter." The role of symmetries and their related conservation laws to scale invariance is a topic of considerable current interest [3]. In particular, Obukhov argued that scale invariance is a natural property for systems in which infinitesimal symmetries lead to Goldstone modes in many body degenerate states [4].

The connection between conservation laws and scale invariance has been tested both experimentally and numerically for nematic liquid crystals (NLC). Fluctuations in NLC are unique in that they vary from microscopic to macroscopic length scales. The slow, long wavelength modes are limited by the size of the sample.

NLC are arrangements of approximately parallel molecules whose centers of mass have no long-range order, similar to ordinary liquids [5]. Interactions between the molecules are invariant under simultaneous rotation of the axis of the molecules and of their center of mass. The total angular momentum is conserved and the components of their molecular quadrupole moment, the director, are the "symmetry breaking" and the "symmetry restoring" variables [6]. Therefore, director fluctuations correspond to the dynamical mode which is critical for all temperatures in the nematic phase [7].

In zero external field, the ordered state has uniform director throughout the sample, $\mathbf{n}=\mathbf{n}_{\mathbf{0}}=$ const. The molecular quadrupole moments couple to external magnetic fields. In strong fields, this coupling leads to a transition from the state with uniform director to a state in which the director varies in space (Fréedericksz transition) [5]. However, in this paper, we are concerned with the behavior of director fluctuations in zero and moderate fields only.

In a recent Letter, Zhang et al. [8] have reported results from neutron scattering experiments on a deuterated NLC ( $d$-PAA). The intensity of the neutron signal is given by the coherent cross section for neutrons scattered by a single molecule which depends on the angle between the director of the molecule and the scattering wave vector. Hence, fluctuations in the recorded neutron signal represent long wavelength orientational fluctuations.

From equilibrium time series, the range $R$ and the standard deviation $S$ of the stochastic variable are obtained. Averaged over the time range $\tau$, the ratio $R / S$ scales with $\tau$ as $R / S \propto \tau^{H}$, where $H$ is the Hurst exponent with $0 \leq H \leq 1$. In the case where successive increments of the stochastic variable are independent of each other, ordinary Brownian motion follows with $\boldsymbol{H}$ $=\frac{1}{2}$. For $H \neq \frac{1}{2}$, which is the case for fractional Brownian motion [9], power-law correlations exist. For $0<H<\frac{1}{2}$, the stochastic process spreads more slowly than diffusively, while for $\frac{1}{2}<H<1$ it spreads more rapidly than diffusively.

Zhang et al. [8] find that the Hurst exponent for director fluctuations changes continuously from $H=1$ in zero field to $H \simeq \frac{1}{2}$ in moderate fields. Such behavior implies long-lived correlations of director fluctuations [10] that persist even in the presence of weak external magnetic fields.

In this paper, we describe director fluctuations in nematic liquid crystals. We calculate their mean square displacements and find that director fluctuations are fractional Brownian processes with a Hurst exponent $H=\frac{3}{4}$ in zero field. Rotational invariance is destroyed by arbitrarily weak magnetic fields, and we find $H=\frac{1}{2}$ for nonzero fields. We emphasize that although we use the language applicable to NLC, our results apply to more general situations.

In nematic liquid crystals, the (average) molecular axis, the director, has the same orientation throughout the sample. For such a uniaxial system, the order parameter is a second rank tensor $S_{\alpha \beta}=n_{\alpha} n_{\beta}-\frac{1}{3} \delta_{\alpha \beta}$. The director may vary in space, $\mathbf{n}=\mathbf{n}(\mathbf{r})$, due to boundary conditions or external fields. The free energy of the NLC has contributions due to elastic distortions and external magnetic fields which may be found, e.g., in Ref. [5]. The dynamic of the director is overdamped, $\eta \partial \mathrm{n} / \partial t=-\delta \mathcal{F} / \delta \mathrm{n}$, where $\eta$ is an effective viscosity.

It is generally assumed that director fluctuations are 
small compared to the equilibrium value, $n=n_{0}+\delta n$ with $\left|\mathbf{n}_{\mathbf{0}}\right| \gg|\delta \mathbf{n}|$, so that fluctuations are orthogonal to the equilibrium director orientation, $\mathbf{n}_{0} \perp \delta \mathbf{n}$. Hence, in a Cartesian coordinate system whose $z$ axis is aligned with $\mathbf{n}_{0}$, the director follows $\mathbf{n}=(0,0,1)+\left(\delta n_{x}, \delta n_{y}, 0\right)$.

We introduce the Fourier transformation of director fluctuations,

$$
\delta \mathbf{n}(\mathbf{r})=V^{-1 / 2} \sum_{\mathbf{q}} \delta \mathbf{n}(\mathbf{q}) e^{-i \mathbf{q} \cdot \mathbf{r}},
$$

where $V$ is the volume of the sample. The free energy is given in Fourier representation by $\mathcal{F}=\frac{1}{2} \sum_{\mathbf{q}}\left[K q^{2}+\kappa h^{2}\right]$ $\times|\delta \mathbf{n}(\mathbf{q})|^{2}$, where $K$ is an elastic constant, $h$ is the magnetic field in the plane orthogonal to the average director $\mathbf{n}_{0}$, and $\kappa$ is the magnetic susceptibility. The equations of motion of the Fourier modes follow as

$$
\eta \frac{\partial}{\partial t} \delta n_{i}(\mathbf{q}, t)=-\left[K q^{2}+\kappa h^{2}\right] \delta n_{i}(\mathbf{q}, t), \quad i=x, y .
$$

Thus, the correlation functions decay exponentially,

$$
\left\langle\delta n_{i}(\mathbf{q}, t) \delta n_{j}\left(\mathbf{q}^{\prime}, 0\right)\right\rangle=\delta_{\mathbf{q q}} \delta_{i j}\left\langle\left|\delta n_{i}(\mathbf{q}, 0)\right|^{2}\right\rangle e^{-t / \tilde{\tau}_{\mathbf{q}}},
$$

where the relaxation time is given by $\tilde{\tau}_{\mathbf{q}}=\eta\left[K q^{2}\right.$ $\left.+\kappa h^{2}\right]^{-1}$. In thermal equilibrium, at temperature $T$, director fluctuations obey the canonical distribution, $\exp \left(-\mathcal{F} / k_{B} T\right)$, and the mean square amplitude of Fourier modes follows as $\left\langle\left|\delta n_{i}(\mathrm{q})\right|^{2}\right\rangle_{\mathrm{eq}}=k_{B} T\left(K q^{2}\right.$ $\left.+\kappa h^{2}\right)^{-1}$, for $i=x, y$. Since $n_{x}$ and $n_{y}$ are equivalent, we set $n_{x}=n$ in the remainder of this paper.

We consider first the behavior of director fluctuations in nonzero external fields, $h \neq 0$. Inserting Eq. (2) into Eq. (1), we find for the correlation function

$$
\langle\delta n(\mathbf{r}, t) \delta n(\mathbf{r}, 0)\rangle=e^{-t / \tau_{h}} \sum_{\mathbf{q}}\left\langle|\delta n(\mathbf{q}, 0)|^{2}\right\rangle e^{-\eta^{-1} K q^{2} t}
$$

with the relaxation time

$$
\tau_{h}=\eta / \kappa h^{2} \text {. }
$$

We neglect slow relaxation processes so that

$$
\sum_{\mathbf{q}}\left\langle|\delta n(\mathbf{q}, 0)|^{2}\right\rangle \exp \left(-\eta^{-1} K q^{2} t\right) \simeq\left\langle[\delta n(\mathbf{r}, 0)]^{2}\right\rangle .
$$

Thus, $\delta n(\mathbf{r}, t)$ is uncorrelated to its initial value $\delta n(\mathbf{r}, 0)$ for times $t>\tau_{h}$ :

$$
\langle\delta n(\mathbf{r}, t) \delta n(\mathbf{r}, 0)\rangle=e^{-t / \tau_{h}}\left\langle|\delta n(\mathbf{r}, 0)|^{2}\right\rangle .
$$

The decay of correlation function is caused by equilibrium fluctuations which can be described by the stochastic "force" $\xi(t)$,

$$
\delta n(\mathbf{r}, t)-\delta n(\mathbf{r}, 0)=\int_{0}^{t} \xi(s) d s .
$$

The stochastic forces $\xi(t)$ and $\xi(s)$ are uncorrelated for $t-s>\tau_{h}$. It follows that the spectral density $G(f)$ $=2 \int_{-\infty}^{\infty} \cos (2 \pi f t)\langle\xi(t+s) \xi(s)\rangle d t$ is constant except for very high frequencies $f>\tau_{h}^{-1}$. We neglect correlations that decay on time scales of the order of $\tau_{h}$, and thus set
$G(f)=$ const so that $\xi(t)$ and $\xi(s)$ are independent of each other for $t \neq s$. Indeed, it has been shown that a Gaussian process is Markovian only when the correlation functions decay exponentially [11]. We thus have

$$
\langle\xi(t) \xi(s)\rangle \sim \delta(t-s) .
$$

The mean square displacement then follows as

$$
\left\langle[\delta n(\mathbf{r}, t)-\delta n(\mathbf{r}, 0)]^{2}\right\rangle=2 \int_{0}^{t} d s \int_{0}^{s} d u\langle\xi(s) \xi(u)\rangle
$$

so that

$$
\left\langle[\delta n(\mathbf{r}, t)-\delta n(\mathbf{r}, 0)]^{2}\right\rangle \sim t
$$

In the absence of external magnetic fields, $h=0$, the relaxation time $\tau_{h}$ diverges, and slow hydrodynamic modes are responsible for the decay of correlation functions,

$$
\tau_{\mathbf{q}}=\eta / K q^{2} .
$$

We find that slow modes have large variance,

$$
\left\langle|\delta n(\mathbf{q})|^{2}\right\rangle_{\mathrm{eq}}=k_{B} T / K q^{2} .
$$

Equations (8) and (9) together with Eq. (1) suggest a hierarchical picture of changes in $\delta n(\mathbf{r}, t)$. Modes with large wave vector produce fast changes which are small in magnitude, while modes with smaller wave vector produce larger changes which are more infrequent.

The elastic approximation breaks down for length scales that are comparable to the (average) distance between neighboring molecules $l$. This length scale introduces a short time scale $\tau_{l}=\left(4 \pi^{2} K\right)^{-1} \eta l^{2}$. We consider times that are longer than $\tau_{l}, t>\tau_{l}$. The size of the sample introduces the length scale $L=V^{1 / 3}$ which, in turn, defines a long time scale $\tau_{L}=\left(4 \pi^{2} K\right)^{-1} \eta L^{2}$. For times $t<\tau_{L}$, surface contributions to the free energy can be neglected.

For finite times, $\tau_{l}<t<\tau_{L}$, the correlation function is given in the continuum limit by

$$
\begin{aligned}
\langle\delta n(\mathbf{r}, t) \delta n(\mathbf{r}, 0)\rangle= & 4 \pi^{2} k_{B} T K^{-1} \\
& \times \int_{2 \pi / L}^{2 \pi / l} \exp \left(-K \eta^{-1} q^{2} t\right) d q .
\end{aligned}
$$

Scaling behavior follows in the limit as $l \rightarrow 0$ and $L \rightarrow \infty$,

$$
\langle\delta n(\mathbf{r}, t) \delta n(\mathbf{r}, 0)\rangle \sim 1 / \sqrt{t} .
$$

That is, in zero field, correlations persist on all time scales.

Equilibrium fluctuations are described by the stochastic "force" $\zeta(t)$,

$$
\delta n(\mathbf{r}, t)-\delta n(\mathbf{r}, 0)=\int_{0}^{t} \zeta(s) d s .
$$

Because the system is linear, the stochastic force is a linear superposition $\zeta(t)=\sum_{\mathbf{q}} \zeta_{\mathbf{q}}(t)$, where the stochastic forces $\zeta_{q}(t)$ and $\zeta_{q^{\prime}}(t)$ are independent of each other for $\mathbf{q} \neq \mathbf{q}^{\prime},\left\langle\zeta_{\mathbf{q}}(t) \zeta_{\mathbf{q}^{\prime}}(s)\right\rangle=\delta_{\mathbf{q} \mathbf{q}^{\prime}}\left\langle\zeta_{\mathbf{q}}(t) \zeta_{\mathbf{q}}(s)\right\rangle$. Correlation of the 
stochastic force $\zeta_{\mathbf{q}}$ decay on time scales of the order of $\tau_{\mathbf{q}}$, i.e., $\left\langle\zeta_{\mathbf{q}}(t) \zeta_{\mathbf{q}}(s)\right\rangle \bumpeq 0$ for $t-s>\tau_{\mathbf{q}}$, and the corresponding spectral density is a constant for small frequencies $f$ $<\tau_{\mathbf{q}}^{-1}$. Since $\tau_{\mathbf{q}}^{-1} \rightarrow 0$ for $|\mathbf{q}| \rightarrow 0$, the spectral density of the fluctuating force $\zeta(t)$ is not a constant even for small frequencies, and thus long time tailed correlations exist. It follows that a Markovian assumption for the stochastic force is no longer valid.

We invoke Onsagers regression hypothesis [12] to find the correlation function of the stochastic force $\zeta(t)$. It states that the correlation of the fluctuating variable at times $t$ and 0 in an equilibrium system, i.e., $\langle\zeta(t) \zeta(0)\rangle$, is the same as the average of the fluctuating variable at time $t$ given that a certain fluctuation occurred at time 0 , i.e., $\langle\delta n(r, t) \delta n(r, 0)\rangle$. We thus find

$$
\langle\zeta(t) \zeta(s)\rangle \sim 1 / \sqrt{t-s} .
$$

The mean square displacement is given by $\langle[\delta n(r, t)$ $\left.-\delta n(\mathbf{r}, 0)]^{2}\right\rangle=2 \int_{0}^{t} d s \int_{0}^{s} d u\langle\zeta(s) \zeta(u)\rangle$. Inserting Eq. (12), we find superdiffusive behavior,

$$
\left\langle[\delta n(\mathrm{r}, t)-\delta n(\mathrm{r}, 0)]^{2}\right\rangle \sim t^{3 / 2} .
$$

The Hurst exponent $H$ is related to the asymptotic behavior of the mean square displacement by $\langle[\delta n(\mathbf{r}, t)$ $\left.-\delta n(r, 0)]^{2}\right\rangle \sim t^{2 H}$ [9]. From Eqs. (7) and (13), we thus find $H=\frac{1}{2}$ for director fluctuations in the presence of external magnetic fields and $H=\frac{3}{4}$ in zero field. Any nonzero external field introduces a time scale $\tau_{h}$ over which correlations decay, and ordinary Brownian motion follows for long times. Thus, director fluctuations in zero and nonzero external magnetic fields belong to two different universality classes.

The Hurst exponent of director fluctuations has been reported for moderate magnetic fields in Ref. [8]. The study is based on analysis of time series with resolution of $20 \mathrm{sec}$ that is recorded over a time range $\tau \sim 11 \mathrm{~h}(2000$ channels). The Hurst exponent is found to vary continuously from $H=1$ in zero field to $H \simeq \frac{1}{2}$ in fields of moderate strength $(\sim 200 \mathrm{Oe})$. This interpretation contradicts our theoretical prediction. However, earlier neutron scattering experiments on $d$-PAA have found $H=\frac{3}{4}$ in zero field [13]. For weak fields, the relaxation time $\tau_{h}$ is of the order of minutes or longer, and ordinary Brownian motion sets in only for long times. In the time series reported in Ref. [8], the chosen time range $\tau$ is not long enough to record the asymptotic behavior of director fluctuations in nonzero fields.

We summarize our results. In nematic liquid crystals, equilibrium director fluctuations are ordinary Brownian processes only in the presence of external magnetic fields. In zero field, long-lived correlations exist, and the mean square displacement increases in time faster than linearly. Such superdiffusive behavior is realized in random walks with long excursions ("Levy walks") [14].

I would like to thank Professor R. Silbey for his hospitality at MIT which made the completion of this work possible.

[1] P. Bak, C. Tang, and K. Wiesenfeld, Phys. Rev. Lett. 59, 381 (1987); Phys. Rev. A 38, 364 (1988).

[2] T. Hwa and M. Kardar, Phys. Rev. A 45, 7002 (1992).

[3] L. P. Kadanoff, Physica (Amsterdam) 163A, 1 (1990).

[4] S. P. Obukhov, Phys. Rev. Lett. 65, 1395 (1990).

[5] P. G. de Gennes, The Physics of Liquid Crystals (Clarendon, Oxford, 1974).

[6] See, e.g., D. Forster, Hydrodynamic Fluctuations, Broken Symmetry, and Correlation Functions (AddisonWesley, Reading, MA, 1990).

[7] P. C. Hohenberg and B. I. Halperin, Rev. Mod. Phys. 49, 435 (1977).

[8] Z. Zhang, O. G. Mouritsen, K. Otnes, T. Riste, and M. J. Zuchermann, Phys. Rev. Lett. 70, 1834 (1993).

[9] B. B. Mandelbrot, The Fractal Geometry of Nature (Freeman, San Francisco, 1982).

[10] R. F. Blundell and A. J. Bray, Phys. Rev. A 46, R6154 (1992).

[11] M. C. Wang and G. E. Uhlenbeck, Rev. Mod. Phys. 17, 323 (1945); reprinted in Selected Papers on Noise and Stochastic Processes, edited by N. Wax (Dover, New York, 1954).

[12] See, e.g., L. E. Reichl, A Modern Course in Statistical Physics (University of Texas Press, Austin, 1987).

[13] K. Otnes and T. Riste, Phys. Scr. T44, 77 (1992).

[14] E. W. Montroll and M. F. Schlesinger, in Nonequilibrium Phenomena II: From Stochastics to Hydrodynamics, Studies in Statistical Mechanics Vol. II, edited by J. L. Lebowitz and E. W. Montroll (North-Holland, Amsterdam, 1984); M. F. Schlesinger, G. M. Zaslavsky, and J. Klafter, Nature (London) 363, 31 (1993). 